\title{
Application of Artificial Intelligence in Mechanical Engineering
}

\author{
Qi Huang \\ School of Electrical and Electionic Engineering, Chongqing University of Technology, Chongqing \\ 400054, China
}

13983308672@163.com

Keywords: artificial intelligence, mechanical and electrical engineering, machine learning, neural network, fault diagnosis.

\begin{abstract}
With the development of computer technology, the application of artificial intelligence technology is more and more extensive. This paper summarizes the artificial intelligence technology, including its development process, composition. What's more, the concept of mechanical and electronic engineering is introduced and the relationship between mechanical and electronic engineering and artificial intelligence technology is analyzed. Finally, the development of artificial intelligence in mechanical fault diagnosis is summarized. Taking the fault diagnosis of hot forging press as an example to illustrate the specific application of artificial intelligence in mechanical engineering.
\end{abstract}

\section{Introduction}

Artificial intelligence is an emerging technology science that studies and develops the theory, technology and application systems for simulating and extending human intelligence, involving disciplines such as psychology, cognitive science, thinking science, information science, systems science and bioscience. The Artificial intelligence is in fact the simulation of the process of data interaction of human thinking, hoping to understand the essence of human intelligence and then produce a smart machine, this intelligent machine can be the same as human thinking to respond and deal with the problem [1].

With the continuous progress of science and technology, mechanical engineering is also constantly evolving and changing, from the traditional mechanical engineering to the electronic mechanical engineering. And its level of automation and intellectualization has a continuous improvement, it went into a new stage of development, thus, the combination of artificial intelligence technology and mechanical and electronic engineering has become a hotspot.

Artificial intelligence technology is applied under the premise of the development of computer technology, which improved the computer technology through the analysis of it to achieve the realization of intelligent technology. When Intelligent technology being applied in mechanical and electrical engineering, it mainly achieved the automation control of mechanical engineering, the applications of artificial intelligence in mechanical and electrical engineering is not only the use of computer technology, but also combined with information technology, psychology, linguistics and other knowledge [2].

The purpose of this paper is to report the composition and development of artificial intelligence, as well as the relationship between artificial intelligence and mechanical and electronic engineering. Most importantly, it aims to study how artificial intelligence is applied in the field of mechanical and electrical engineering.

\section{The Research Direction of Artificial Intelligence}

\subsection{Machine Learning}

Machine Learning (ML), which mainly focuses on how the computer simulates human learning behavior, reorganizes the existing knowledge structure with the knowledge and skills learned, and continuously improves its performance. Machine learning is the core of artificial intelligence and it is 
the only way for computers to have its own intelligence. At present, the machine learning is used in all areas of artificial intelligence, but cannot be used for deductive reasoning [3].

\subsection{Expert System}

Expert system (ES) is another important branch of artificial intelligence research [4]. It will explore the general way of thinking into the use of specialized knowledge to solve specific problems. ES will make the theoretical research of the artificial intelligence into practical application; expert system can be seen as a kind of specialized knowledge of computer intelligent program system, it can use expertise and experience provided by experts in specific areas and the use of reasoning techniques in artificial intelligence to solve and simulate complex problems that can often be solved by experts. A basic expert system consists of knowledge base, database, reasoning machine, interpretation mechanism, knowledge acquisition and user interface, as shown in Figure 1.

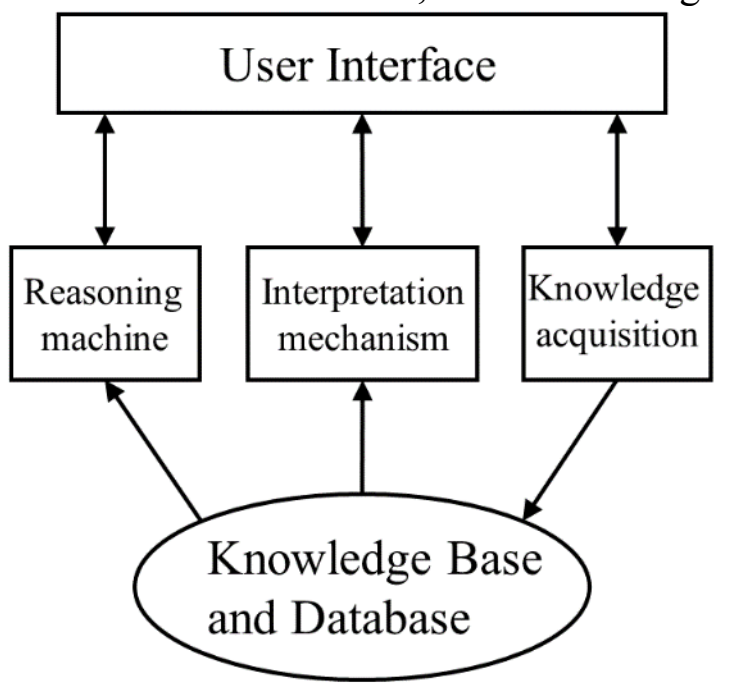

\subsection{Pattern Recognition}

Fig. 1 The basic structure of the expert system

Pattern recognition research mainly includes two aspects: one is the method of perception of the object, which belongs to the understanding of scientific category; the other one is to achieve pattern recognition with the computer under the condition of the task of the case is determined. The former is the research content of physiologists, psychologists, biologists and neurophysiologists. The latter has been systematically researched by the efforts of mathematicians, informatics experts and computer science workers, and has been applied in text recognition, voice recognition, fingerprint identification, remote sensing and medical diagnosis, etc., it has greatly facilitated the people's lives.

\subsection{Neural Network}

Artificial Neural Network (ANN) is an arithmetic model obtained by abstracting the human neural network from the perspective of information processing. It is composed of a large number of neurons connected with each other. Each neuron represents a specific output function, called an excitation function. The connection between each of the two neurons represents a weighting through the connected signal, called the weight. When the neural network connection mode, weight and incentive function changes, the network output also will change [5].

\subsection{Deep Learning}

The concept of deep learning comes from artificial neural network research, belonging to a new field of machine learning [6]. Depth learning refers to artificial intelligence beginning to learn, train it, self-master concepts, and recognize sounds, images and other data from untagged data. This approach is closer to the human brain. Deep learning is mainly to build a deep structure to learn multi-level representation, not specifically refers to a machine learning algorithm or model, but a technology. 


\section{The Concept of Mechanical and Electronic Engineering}

Mechanical and electrical engineering is a science and technology covering all kinds of science, the core of which is mechanical electronics, combined with related knowledge of information technology and intelligent network. The theory of these disciplines has been widely used in mechanical and electrical engineering. In the design of mechanical and electrical engineering, it is necessary to integrate the computer technology, network technology and mechanical-related technology, combining the different mechanical components to improve the design. Although the knowledge is very complex in the design of the mechanical and electronic engineering, the design is relatively simple, the structure is not complicated, and has good performance. Mechanical and electronic engineering has high efficiency, small size when it went to production, which replaced the traditional machinery [7].

\section{The Relationship between Artificial Intelligence and Mechanical and Electronic Engineering}

With the rapid development of electronic information, mechanical and electronic engineering as a basic discipline has been widely used in our life. But the mechanical and electronic engineering also has shortcomings, such as the unstable system, the reason of the problem is the imperfect factor of the electronic information system. Artificial intelligence itself can quickly transfer the information and timely process it, which can effectively make up for this shortcoming. In the process of input and output in mechanical and electronic engineering, the electronic information system will face a lot of difficulties and resistance, if the input information is too complex, electronic information system is likely to make a mistake, then you need to manually solve the problem. If you can combine the two features, you can solve the problems and shortcomings of mechanical and electrical engineering.

\section{Application of Artificial Intelligence in Mechanical Engineering}

At present, artificial intelligence technology is often used in the diagnosis of mechanical engineering failure [8-10]. In general, artificial intelligence-based fault diagnosis techniques include rule-based reasoning (RBR), case-based reasoning (CBR), and fault-based tree fault diagnosis.

Based on the basic composition and basic principle of the traditional expert system, a mechanical fault diagnosis expert system based on RBR and CBR reasoning is constructed. The overall structure is shown in Figure 2. The system includes maneuver case database, fault diagnosis rule database, fault diagnosis database, fault reasoning machine, knowledge processing, fault diagnosis process interpreter, learning system and expert system man-machine interface.

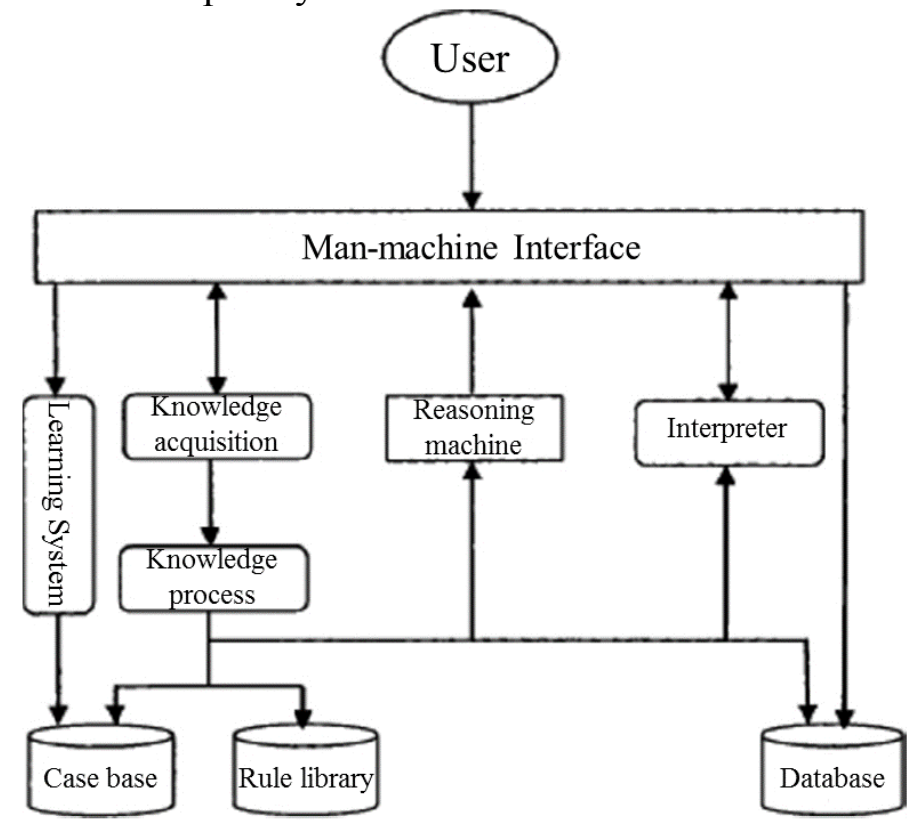

Fig. 2 The overall structure of the system 
The basic working process of the diagnosis system is: Firstly, the user input the online data monitored by the machine through the man-machine interface. Secondly, reasoning machine activate the corresponding rules to obtain diagnostic results according to the positive reasoning mechanism, it will provide diagnostic expert advice, and then retrieve the case in the database through a certain algorithm, subsequently, get the most similar case, calculate the similarity according to the historical case, and complete the mechanical fault diagnosis with high efficiency. Finally, it will further improve the expert diagnosis system by adding new cases.

\subsection{Intelligent Diagnostic System for Rotating Machinery}

From the situation of fault diagnosis of mechanical equipment, during the development of several years, the theory and method of fault diagnosis technology of rotating machinery has been improved day by day. In the practical application, it has achieved great economic benefits. In this paper, the fan diagnosis system is used as an example, in fact, it is the universal integrated neural network diagnosis system in the fan fault diagnosis application.

The system is composed of two parts: fan and motor. According to the type of monitoring parameters, the main system can be divided into five subsystems: vibration, temperature, noise, oil and performance, in which the fault diagnosis and decision system is core of the whole intelligent system.

\subsection{Intelligent Diagnosis System for Reciprocating Machinery}

Because the reciprocating machinery has a set of high-speed reciprocating motion quality, its kinematics and dynamic morphology is much more complex than the rotating machinery, fault diagnosis is more difficult. The research about failure mechanism and diagnostic methods of research is not enough. Diesel engine is a typical reciprocating machine; integrated neural network diagnosis system is actually a universal integrated neural network diagnostic system in the applications of diesel engine fault diagnosis.

Diesel engine failure can be divided into performance failure and mechanical failure. The diagnosis of performance faults can be achieved using a sub-neural network, with performance parameters as input, such as power, speed, cylinder pressure, water temperature and so on. The mechanical fault is diagnosed by two sub-neural networks, and the integrated neural network diagnosis system is formed by using the commonly used vibro acoustic (VA) signal and oil analysis information as input.

\subsection{Application of Fault Diagnosis to Hot Film Forging Press}

In the process of production of hot forging presses, it will produce some common serious problems, such as the slider stopped outside the location of the top dead center, stuffy car, the main motor current is too high, lubrication failure. There are many reasons for these failures, and the fault diagnosis method of hot forging press can be combined with rule reasoning and case reasoning [11]. The fault diagnosis of the production process of the hot forging press is based on the rule reasoning and the case reasoning. The system carries out the relevant rule reasoning and case reasoning according to the case in the case library and establishes the number of failures. And the nearest neighbor algorithm is used to analyze the similarity of case matching. The algorithm is as follows:

$$
\operatorname{sim}(X, A)=\sum_{i=1}^{n} w_{i} \operatorname{sim}_{i}\left(f_{i}^{X}, f_{i}^{A}\right) / \sum_{i=1}^{n} w_{i},
$$

Where, $w_{i}$ is the weight of the $i^{\text {th }}$ attribute, $f_{i}^{X}$ represents the value of the $i^{\text {th }}$ attribute of the fault $X, f_{i}^{A}$ represents the value of the $i^{\text {th }}$ attribute of the case $A, \operatorname{sim}_{i}\left(f_{i}^{X}, f_{i}{ }^{A}\right)$ is the similarity of the fault $X$ and the retrieval case $A, \sum_{i=1}^{n} w_{i}=1$. Different types of attribute values, the calculation method of $\operatorname{sim}_{i}\left(f_{i}^{X}, f_{i}^{A}\right)$ is not the same:

(1) The value of the attribute of the string type is calculated by the TF-IDF method. The similarity is expressed by the cosine of the angle of the weight vector, which is shown in Equations (2) and (3): 


$$
\begin{aligned}
& w_{i}=f_{i} \times \lg \left(\frac{N}{N_{j}+1}\right), \\
& \operatorname{sim}(X, A)=\frac{\sum_{j=1}^{t}\left[f_{X j} \times \lg \left(\frac{N_{X}}{N_{X j}+1}\right)\right]\left[f_{A j} \times \lg \left(\frac{N_{A}}{N_{A j}+1}\right)\right]}{\sqrt{\sum_{j=1}^{t}\left[f_{X j} \times \lg \left(\frac{N_{X}}{N_{X j}+1}\right)\right]^{2}} \sqrt{\sum_{j=1}^{t}\left[f_{A j} \times \lg \left(\frac{N_{A}}{N_{A j}+1}\right)\right]^{2}}},
\end{aligned}
$$

Where, $j$ represents the attribute of string type, $j=1, \cdots, t, f_{j}, f_{X j}$ and $f_{A j}$ is the frequency of the fault; $N, N_{X}$ and $N_{A}$ represent the total number of string type attributes in the case; $N_{j}, N_{X j}$ and $N_{A j}$ represent the number of occurrences of a string type attribute in the case.

(2) For the value type attribute is the range of $[a, b]$, the calculation of $\operatorname{sim}_{i}\left(f_{i}^{X}, f_{i}^{A}\right)$ is as follows:

$$
\operatorname{sim}_{i}\left(f_{i}^{X}, f_{i}^{A}\right)=1-\frac{\left|f_{i}^{X}, f_{i}^{A}\right|}{b-a} ; f_{i}^{X}, f_{i}^{A} \in[a, b],
$$

The results of case matching are listed in the similarity degree from large to small. The design engineer uses the larger similarity fault diagnosis to design scheme, and sets a similarity threshold $\delta$ to improve the diagnosis efficiency.

\section{Conclusion}

This paper reviews the composition and development of artificial intelligence, as well as the relationship between artificial intelligence and mechanical and electrical engineering. It also summarizes the relevant applications in the field of mechanical engineering. Theoretical and practical research shows that intelligent technology has been widely used in all aspects of mechanical systems, coupled with the discovery of knowledge and distributed artificial intelligence and other computer technology, which makes artificial intelligence more effective in the mechanical system and other areas. Because of the increasingly fierce competition in the machinery industry, the hybrid intelligent design, monitoring, control, diagnosis system based on fuzzy logic, neural network, expert system, will be a new research hotspot in order to improve the level of its intelligent control. These applications have very promising prospects.

\section{References}

[1] P Norvig, SJ Russell. Artificial intelligence: a modern approach [J]. Applied Mechanics \& Materials, 2003, 263 (5): 2829-2833.

[2] RA Brooks. Intelligence without representation [J]. Artificial Intelligence, 1991, 47 (1-3): 139-159.

[3] DE Goldberg, JH Holland. Genetic algorithms and machine learning [J]. Machine Learning, 1988, 3 (2): 95-99.

[4] B Chandrasekaran. Generic tasks in knowledge-based reasoning: High-level building blocks for expert system design [J]. IEEE Expert, 1986, 1 (3): 23-30.

[5] MH Hassoun. Fundamentals of artificial neural networks [J]. Proceedings of the IEEE, 1996, 84 (6): 906.

[6] Y Lecun, Y Bengio, G Hinton. Deep learning [J]. Nature, 2015, 521 (7553): 436-444.

[7] W Bolton. Mechatronics electronic control systems in mechanical and electrical engineering [J]. Fish Physiology \& Biochemistry, 2009, 35 (3): 385-398.

[8] H Yang, J Mathew, L Ma. Intelligent diagnosis of rotating machinery faults - A review [M]. Pattern Recognition \& Data Mining, 2002 
[9] LB Jack, AK Nandi. Fault detection using support vector machines and artificial neural networks, augmented by genetic algorithms [J]. Mechanical Systems \& Signal Processing, 2002, 16 (2-3): 373-390.

[10] A Siddique, GS Yadava, B Singh. Applications of artificial intelligence techniques for induction machine stator fault diagnostics: review [J]. IEEE International Symposium on Diagnostics for Electric Machines, 2003, 49 (3): 29-34.

[11] WH Wen. Application of artificial intelligence technology in mechanical and electronic engineering $[\mathrm{J}]$. Automation and instrumentation, 2016, 2: 96-97. 